\title{
Local brightness mechanisms sketch out surfaces but do not fill them in: Psychophysical evidence in the Kanizsa square
}

\author{
BIRGITTA DRESP \\ Laboratoire de Psychologie Expérimentale, CNRS, Université Paris V, Paris, France
}

\begin{abstract}
In two experiments, brightness enhancement of the illusory surface in the Kanizsa square was investigated by means of a brightness matching procedure. The results show that specific properties of the inducing elements such as size, spacing, and luminance have effects on the matching threshold that are similar to those previously obtained in experiments on simultaneous contrast. The data from a third experiment demonstrate that increment thresholds measured within the Kanizsa square are elevated when the target is flashed on a position close to the inducing elements. The thresholds decrease considerably in the center of both test and control figures (representing or not representing an illusory square). These observations suggest that low-level mechanisms are likely to explain local brightness differences within the configurations but not global figure brightness. In other words, local contrast seems to generate brightness information that "sketches out" surfaces at their surrounds but does not "fill" them "in."
\end{abstract}

Whether illusory figures such as the Kanizsa square (Kanizsa, 1955) are "curious cases of simultaneous brightness contrast”' (Frisby \& Clatworthy, 1975; see also Brigner \& Gallagher, 1974) is an old question that has never really been settled. The few attempts (see, e.g. Spillmann, Fuld, \& Neumeyer, 1984) to investigate the effects of brightness induction in illusory figures by means of indirect psychophysics (by using procedures other than scaling or rating) have not provided satisfactory estimates of the phenomenon. Direct psychophysical measures, based essentially on magnitude estimation procedures (see, e.g., Dresp, Lorenceau, \& Bonnet, 1990; Watanabe \& Oyama, 1988), have shown that figural brightness (or darkness when the inducing elements are white) in the Kanizsa square increases with the size and the proximity of the inducing elements. These findings, showing how geometric properties of local configurations determine global brightness or darkness, reveal similarities with earlier psychophysical data from experiments on simultaneous contrast patterns (e.g., Heinemann, 1955, 1972).

Using more or less formalized information processing approaches, several authors (Gerrits \& Vendrik, 1970; Grossberg \& Mingolla, 1985; Spillmann, Fuld, \& Gerrits, 1976; Ullmann, 1984) have demonstrated how neural mechanisms, generally described in terms of lateral inhibition, can account for various brightness phenomena. On the basis of their spreading capacity (Gerrits \& Vendrik, 1970), these mechanisms generate a diffusion of brightness or darkness over a more or less large spatial scale.

The author's present address is: Neurologische Klinik der Universität Freiburg, Abteiluug für Neurophysiologie, Hansastrasse 9, Freiburg i. Br., Germany.
Across luminance steps (as present in gratings and similar patterns), such a diffusion gives rise to brightness or darkness gradients, sometimes also called Mach band phenomena. Earlier experiments (see, e.g., Fiorentini, 1972) have demonstrated that locally estimated thresholds for luminance increment detection appear to be sensory correlates of such phenomenal variations, sustaining the idea of a genesis at early stages of visual information processing.

As for surfaces surrounded by areas of opposite luminance, diffusive mechanisms might generate an even spread of brightness or darkness via a process that is quite often referred to as filling in (see, e.g., Gerrits \& Vendrik, 1970; Grossberg \& Mingolla, 1987). A mechanistic description of the filling-in process would be that luminous (or dark) surrounds trigger an inwardly oriented propagation (and summation) of neural signals of differential brightness. In the case of the Kanizsa square, the illusory borders would act as barriers to brightness or darkness propagation in very much the same way as real borders (see, e.g., Grossberg \& Mingolla, 1985).

One of the issues of the study reported here is to provide psychophysical estimates of the parameters that determine the perception of bright or dark surfaces such as the Kanizsa square. Although this figure seems to be a rather particular stimulus, the results of the present investigation indicate that global brightness effects are generated in very much the same way as those produced by configurations with closed surrounds.

A further question is that of the level of processing at which homogeneous surface brightness or darkness arises. If we can provide evidence that a spreading of local contrast mechanisms is likely to generate evenly distributed surface brightness, it will not be necessary to refer to 
higher processes of perceptual organization to account for the perception of illusory and other forms. Whether lowlevel mechanisms provide a satisfactory explanation of these phenomena or whether higher processes of perceptual organization have to be taken into account is a crucial question. In other words, do local brightness and figural brightness arise at the same functional level?

The third experiment in the present study shows variations in thresholds for luminance increment detection observed within a homogeneously dark Kanizsa square. These results indicate that the global intensity of surfaces does not seem to be generated at early stages of processing.

\section{EXPERIMENT 1}

This first experiment was set up to test whether global effects of induced darkness can be measured within the Kanizsa illusion by an indirect psychophysical method. The figures used here consisted of white inducing elements displayed on a dark background. As a consequence, the illusory figure appeared as a gray square on a phenomenally less dark background, the physical luminance of both areas being strictly identical. This situation was chosen for essentially technical reasons. Although the effects with dark inducers on a white background would probably not be symmetrical, there is no reason to doubt that the general tendencies would lead to essentially the same conclusions (see, e.g., Spillmann et al., 1984).

Estimates of darkness induction obtained through a matching procedure might settle the question of whether or not Kanizsa squares are "curious cases of simultaneous contrast." Tasks in which the observer has to make lightness matches between a test and a comparison field by manipulating the luminance of the test field, as well as increment threshold procedures, are widely used to measure the effects of brightness or darkness induction (see Wyszecki, 1986, for a review). The inducing field may increase or decrease the brightness of a test field, depending on the relative luminances of the two stimuli. Geometric parameters such as size and separation of the two fields have a determining influence on induced brightness. Earlier experiments have shown that induction effects increase when the size of the inducing field increases or the distance between the test field and the inducing field decreases (Heinemann, 1955, 1972). In the study described here, observers were asked to manipulate the luminance of Kanizsa squares so that the darkness of the square surface matched the darkness of the background of the figure. If simultaneous contrast effects account for the perception of the illusory square, the induced darkness as reflected through the measures of perceived equality in this experiment should increase when the size of the inducing elements increases and their separation decreases.

\section{Method}

Subjects. Four subjects participated in this experiment. One of them was the author herself. All 4 observers had normal vision, and 3 of them were not aware of the purpose of the experiment.
Materials. The stimuli were presented binocularly on a highresolution video monitor (Visionor Model M 51 CHR No. 1007. Lille, France). They were generated through a PC-compatible computer (Olivetti $M$ 24) with the use of a special graphics adaptor (GALAXY ref. SA-1019A, Evroz, Tel Aviv) that provided a display of $1,024 \times 768$ pixels (horizontally $\times$ vertically) at a $60-\mathrm{Hz}$ frame rate (noninterlaced). The pixel size was $0.33 \times 0.33 \mathrm{~mm}$. The stimuli were 12 versions of the Kanizsa square with varying size and spacing of the four inducing elements (see Figure 1). Four interelement distances were combined with three element sizes. The distances were $20,60,100$, and 140 pixels; the three diameters of the inducing elements were 20,60 , and 100 pixels $(1$ pixel $=$ $0.025^{\circ}$ ). The luminance of the inducing elements was $68 \mathrm{~cd} / \mathrm{m}^{2}$; the luminance of the background, $11 \mathrm{~cd} / \mathrm{m}^{2}$. The gray levels of the test surface were obtained through combinations of RGB signals, carefully calibrated with a CS 100 Minolta photometer.

Procedure. Points of subjective equality (PSE) were measured by means of an adaptive plus or minus procedure derived from Tyler (1987). This staircase method leads to a rapid convergence of the gray values near the asymptotic matching level. Stopping criteria are continuously calculated on the 15 preceding trials according to the following principle: A PSE is reached when the slope of the function relating the matched luminance to the rank of the trial is equal to $0 \pm 1$ and its intercept corresponds to $50 \%$ responses of one category. With this procedure, an average of 50 trials is necessary for measuring one PSE.

The experiment was run in a dark room. Each observer was placed in front of the screen at a viewing distance of about $75 \mathrm{~cm}$ and the head position was stabilized by means of a head- and chinrest. On each trial, the subject had to decide, as fast as possible, whether the surface of a given Kanizsa square was darker or brighter than the background of the figure. "Darker" was followed by an increment, "brighter" by a decrement, in the luminance of the square. The level that was given to start the procedure varied randomly from measure to measure, to ensure that the numbers of increment and decrement trials were balanced.

The observers had to look at the center of the screen, where a white pixel, of weak luminance but clearly visible, indicated the fixation point during the presentation intervals. Responses were recorded by means of two response keys. Presentation time of the figures was about $400 \mathrm{msec}$ ( 25 frames). Within each experimental session, matching thresholds were measured for each of the 12 Kanizsa figures. The presentation order of the figures within each session was randomized. Each subject had to go through five ex-

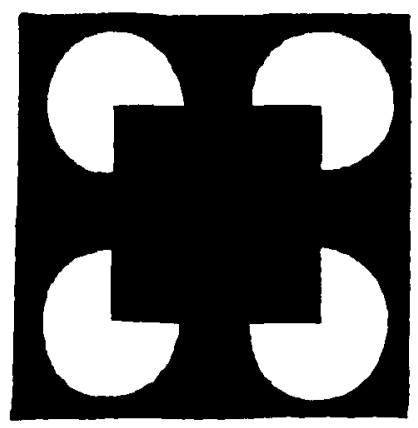

Figure 1. A Kanizsa square with white inducing elements on a dark background. This configuration gives rise to strong darkness enhancement in the center of the square, as demonstrated through direct psychophysical scaling (see Dresp, Lorenceau, \& Bonnet, 1990). 


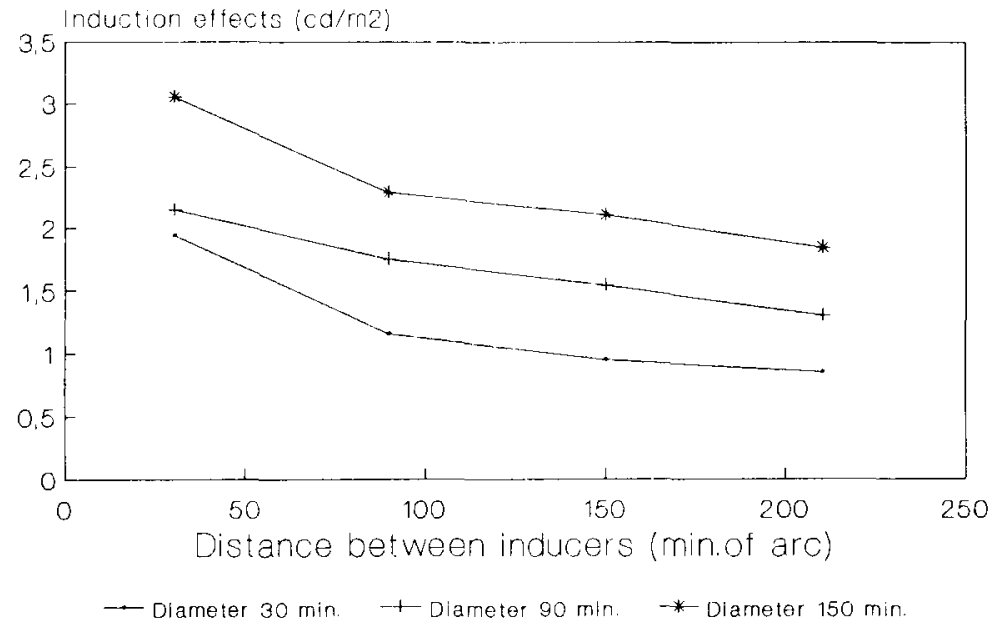

Figure 2. Induction effects, as a function of size and spacing of the inducing elements (Experiment 1). The induction effect expresses the difference between the PSE and the luminance of the comparison field, which was the background of the configurations in this experiment. The curves show that induction effects decrease when the spacing of the inducing elements increases and their diameter decreases.

perimental sessions and was dark adapted at the beginning of each session.

\section{Results and Discussion}

The results of this experiment are shown in Figure 2. The PSEs show that the subjects adjusted the luminance of the test field (the square) to a level higher than the intensity of the comparison field (the background of the figure). Such a result is generally observed when a light inducing field increases the darkness of a test field (Heinemann, 1955, 1972). In the Kanizsa square, the white inducing elements increase the darkness of the square in the center of the figure relative to the darkness of the background. Furthermore, this induction effect increases when the size of the inducing elements increases and their separation decreases. The effect of each factor was statistically significant $[F(2,6)=55.936, p<.05$, and $F(3,9)=36.413, p<.05$, respectively]. The results are consistent with earlier data obtained through crossmodality matching, a more subjective procedure (Dresp et al., 1990).

This experiment shows that the inducing elements of the Kanizsa illusion produce psychophysically measurable effects comparable to those previously observed with stimuli that give rise to simultaneous contrast. In this respect, it provides an answer to an old question that cannot be settled on the basis of phenomenal observation.

The degree to which local darkness enhancement is determined by the luminance of the inducing elements was tested in a second experiment. Earlier studies have shown that the effects of brightness or darkness induction increase with the luminance of the inducing field (Heinemann, 1955, 1972).

\section{EXPERIMENT 2}

Previous experiments in which the observers have had to match the brightness of a test field to the brightness of the illusory surface perceived in the center of the Ehrenstein figure (Spillmann et al., 1984) suggest that significant effects of the luminance of brightness- or darknessinducing elements on the PSE are likely to be obtained at low levels of figure-ground contrast. In the following experiment, at a constant background level, the effect of different intensities was tested, beginning with a luminance at which the inducing elements were just discriminable from the background. It is difficult to make predictions concerning the function that relates the strength of brightness enhancement of a surface to the luminance of the inducing elements. Earlier studies done with the Ehrenstein figure failed to provide satisfactory estimates (see Spillmann et al., 1984).

\section{Method}

Subjects. The subjects were those who participated in Experiment 1.

Materials. The apparatus was that used in Experiment 1. The stimulus in this second experiment was a single Kanizsa square with light inducing elements of varying luminance. The effect of six different intensities on the PSE was tested: 14.40, 19.20, 26.30, 35.70, 48.10 , and $68.30 \mathrm{~cd} / \mathrm{m}^{2}$. The luminance of the background of the figure was held constant at $11 \mathrm{~cd} / \mathrm{m}^{2}$. The diameter of the inducing elements was 100 pixels, and the distance between them at each side of the square, 20 pixels.

Procedure. The same adaptive procedure as that for Experiment 1 was used to measure PSEs. The presentation time of the figures was about $400 \mathrm{msec}$ ( 25 frames). All the intensity levels of the inducing elements were presented within each experimental session. The presentation order of the six different luminances within each 
session was randomized. Each of the 4 observers had to go through five experimental sessions and was dark adapted at the beginning of each session.

\section{Results and Discussion}

The results of this experiment are represented in Figures 3 and 4 . The curves show that the subjective equality level increases as the luminance of the inducing elements increases. Similar observations have been made earlier in simultaneous contrast experiments with a test field surrounded by an annulus inducing field of varying luminance (Heinemann, 1955, 1972). The effect was statistically significant $[F(5,15)=22.014, p<.05]$. A power function was found to provide a satisfactory fit to the experimental data (see Figure 5).
The consistency of the results of this second experiment with data obtained in achromatic induction experiments adds further support to the idea that simultaneous contrast must somehow contribute to the genesis of the illusory phenomenon in the Kanizsa figure. However, the rather global estimates from Experiments 1 and 2 do not allow one to draw conclusions concerning the processes that might explain how global figure enhancement is generated. To decide whether mechanisms such as spreading lateral interactions constitute a satisfactory explanation, data on local information processing are necessary.

Estimates of local sensitivity provided through increment threshold measures may give us useful information about the spatial properties of the mechanisms that can be assumed to be underlying, at least partly, this bright-

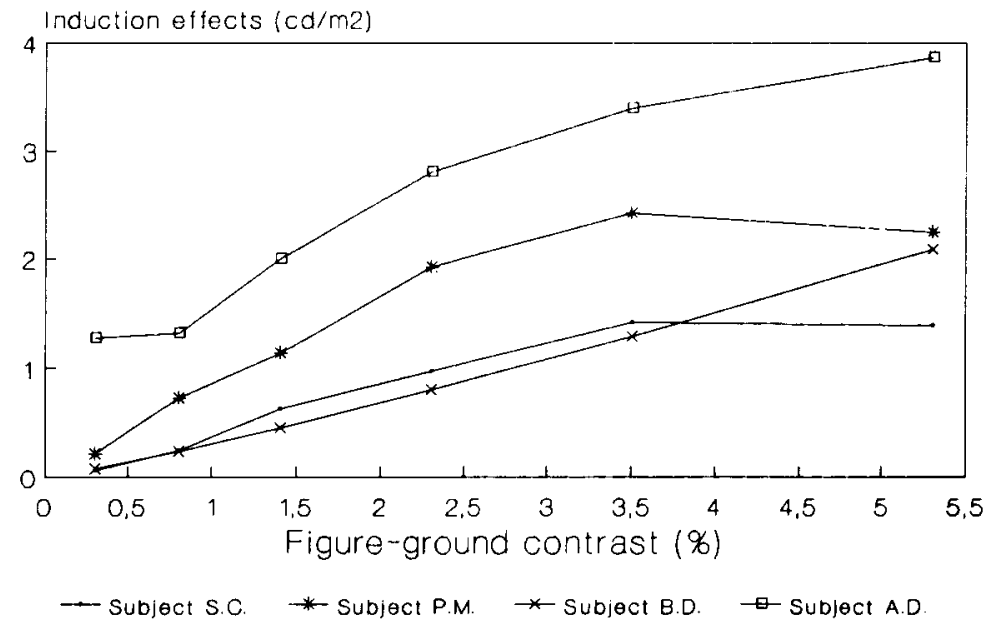

Figure 3. Individual data obtained in Experiment 2. Induction effects tend to increase with increasing figure-ground contrast.

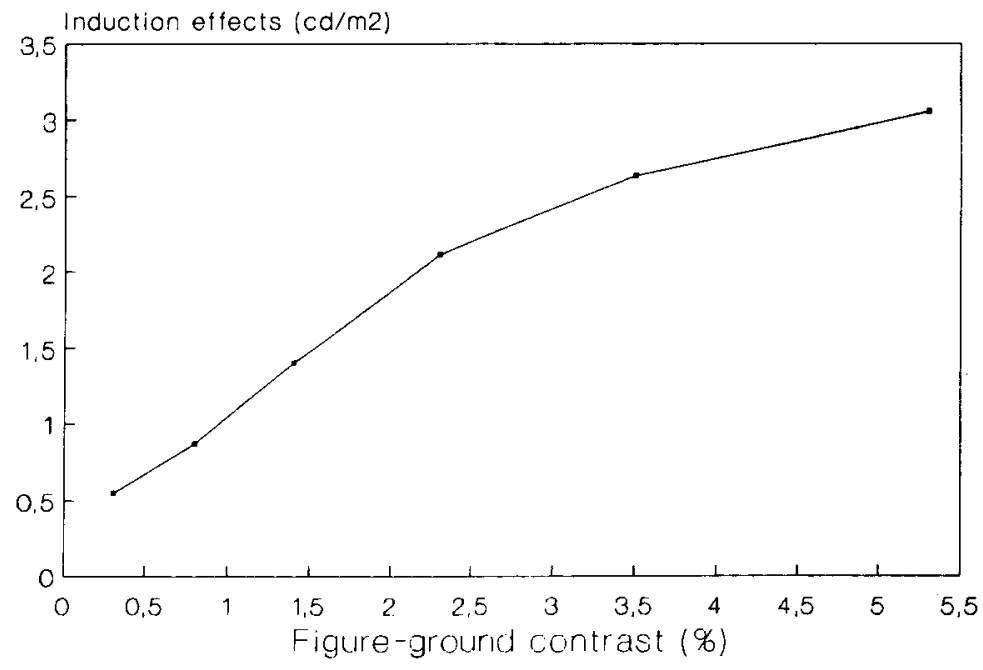

Figure 4. Mean results of Experiment 2. 


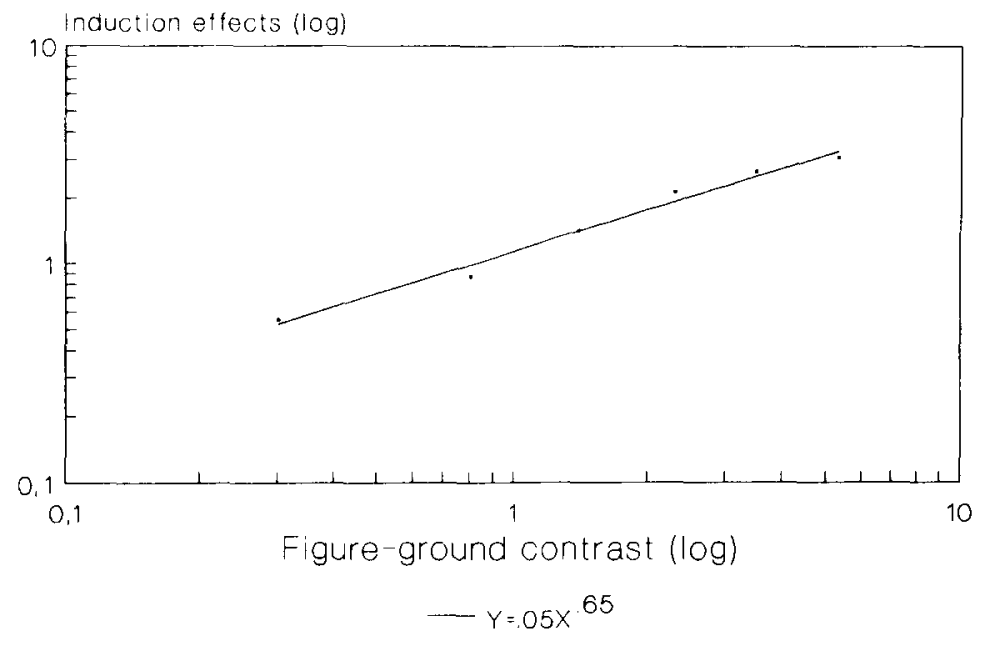

Figure 5. The theoretical function that best represents the data of Experiment 2. Induction effects appear to be related to figure-ground contrast by a power function $(r=.9954)$.

ness phenomenon. In a third experiment, we investigated the effects of induced darkness on increment thresholds measured at different positions within an illusory square and a control configuration without figure enhancement.

\section{EXPERIMENT 3}

Increment thresholds for the detection of a small light spot have been measured before in various stimulus configurations where simultaneous contrast is generated (Cornsweet \& Teller, 1965; Fiorentini, 1972; Jory, 1987; Spillmann et al., 1984; Van Esen \& Novak, 1974). Changes in visual sensitivity, as reflected through increment or decrement threshold variations in dark or bright regions, are assumed to be related to changes in local processing of differential brightness (see Fiorentini, 1972, for a review).

Earlier studies have shown that phenomenally strong brightness or darkness enhancement in the center of surfaces surrounded by inducing fields of opposite luminance does not have a measurable effect on increment thresholds. Such a conclusion was drawn by Cornsweet and Teller (1965), who used a disk surface surrounded by an annulus of opposite intensity, as well as by Van Esen and Novak (1974), who used a square configuration. The latter have reported symmetrical effects, with regard to the sign of contrast, on increment and decrement thresholds. Both studies show that increment thresholds are elevated when the target is located close to the inducing surround and that they tend to decrease when the target is shifted progressively toward the center of the enhanced surface. Recently, Dresp and Bonnet (1991) have observed a progressive decrease of increment thresholds within the Kanizsa square when the target is shifted progressively from the periphery to the center of the illusory square.

If global brightness or darkness enhancement of a surface is generated, by way of some sort of diffusive ac- tivity, at the same level of processing as local variations that can be seen across contrast borders (e.g., Mach bands), why is visual sensitivity not homogeneous within a homogeneously bright or dark surface? Since locally induced brightness or darkness is known to alter systematically decrement or increment thresholds, why do these alterations not correlate with global distributions of subjective intensity perceived within figures?

In the following experiment, increment thresholds were measured in the center of an illusory square and in its periphery, at a position close to the inducing elements. These estimates were compared with measures taken within a control figure where no enhanced surface is perceived. If global darkness is a direct result of low-level routines generating a filling-in process like the one suggested, for example, by Ullmann (1984) or Grossberg and Mingolla (1985), the thresholds should not vary within the Kanizsa square, but they should, however, be systematically altered in comparison with thresholds measured on a plain dark field.

\section{Method}

Subjects. Two highly trained observers participated in this experiment. One was the author herself; the other subject was not aware of the purpose of the experiment. Both observers had normal vision.

Materials. The apparatus was that used in the previous two experiments. The light target (one pixel) that had to be detected by the observers was flashed on the illusory surface of the same Kanizsa square as the one that was used in Experiment 2. In another condition, the inducing elements were turned upside down and no illusory square was perceived (see Figure 6). In both cases, the gap between the inducing elements was 20 pixels, their diameter was 60 pixels, and their luminance was $21 \mathrm{~cd} / \mathrm{m}^{2}$. The dark background was set at $2 \mathrm{~cd} / \mathrm{m}^{2}$. Gray levels of the target pixel were obtained through combinations of RGB signals, carefully calibrated with a CS 100 Minolta photometer.

Procedure. The experiment was run in a dark room, and the observers were dark adapted at the beginning of each session. They 


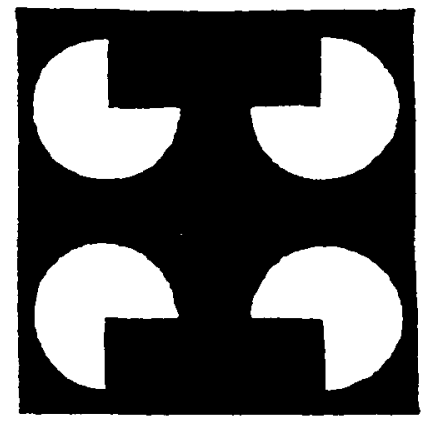

Figure 6. The control configuration used in Experiment 3.

were placed in front of the screen and their head positions were stabilized by means of a head- and chinrest. The illusory figure was exposed continuously in the center of the screen. The exposure time of the target pixel was set at approximately $170 \mathrm{msec}$ ( 10 frames). Increment thresholds were measured in the center of the figures as well as at a position close to the inducing elements (on the horizontal median axis of the figures at a distance of precisely 4 pixels from the illusory contour in the Kanizsa square; the equivalent position was taken in the control configuration). Within each session, at least one threshold was measured on a homogeneous dark background $\left(2 \mathrm{~cd} / \mathrm{m}^{2}\right)$. Each threshold measured within the illusory square was divided through a control threshold obtained on the homogeneous background within the same experimental session. This condition is important to evaluate effects of induced darkness as well as to control possible side effects related to incidental variations of the luminance output of the screen and/or of the visual sensitivity of the observers.

Increment thresholds were measured by means of the same type of adaptive procedure as the one described previously. On each trial, the target pixel could be present or absent with equal probability. The subject gave his/her response ("target seen" or "target not seen") by means of two response keys. Increment or decrement of the target was determined by the subject's response on the pre vious trial: "pixel not seen" was followed by increment, "pixel seen" by decrement. The first five trials of the series served to determine the starting intensity of the target by means of a halving procedure, which permitted the measures to begin as closely as possible to the asymptotic threshold value. After the first trials, the step amplitude was constant (about $0.05 \mathrm{~cd} / \mathrm{m}^{2}$ ). After 15 trials, stopping criteria were calculated from trial to trial. A threshold was obtained when two conditions were met: The slope of the function that related the intensity of the target to the rank of the trial had to be equal to $0 \pm 0.1$, and the percentage of correct detections had to be $75 \pm 10$. An average of 50 trials were necessary to obtain one threshold. At each target position, three thresholds were measured in different sessions. Previous studies (see Dresp \& Bonnet, 1991), with 1 of the observers who participated in this experiment and another subject, have shown that a two-alternative forced-choice procedure leads to essentially the same results as does the adaptive yes/no procedure.

\section{Results and Discussion}

Figures 7 and 8 present the results of this experiment. The graphs show that increment thresholds are elevated (ratio $>1$ ) when the target is flashed at a position close to the inducing elements, which means in the periphery of the illusory square or of the more or less open field in the control figure. The thresholds decrease when the target is presented in the center of the figures. Coefficients of intraindividual variability $(\sigma / \sqrt{N})$ do not exceed .04 for any of the conditions, which indicates that the measures are reliable.

The results of this experiment are consistent with earlier observations reported from the use of stimuli that give rise to enhanced surface brightness or darkness (Cornsweet \& Teller, 1965; Van Esen \& Novak, 1974). Increment thresholds tend to decrease within the center of areas surrounded by fields of opposite luminance.

Regardless of the target position, the threshold alterations are generally higher in the control figure, where no

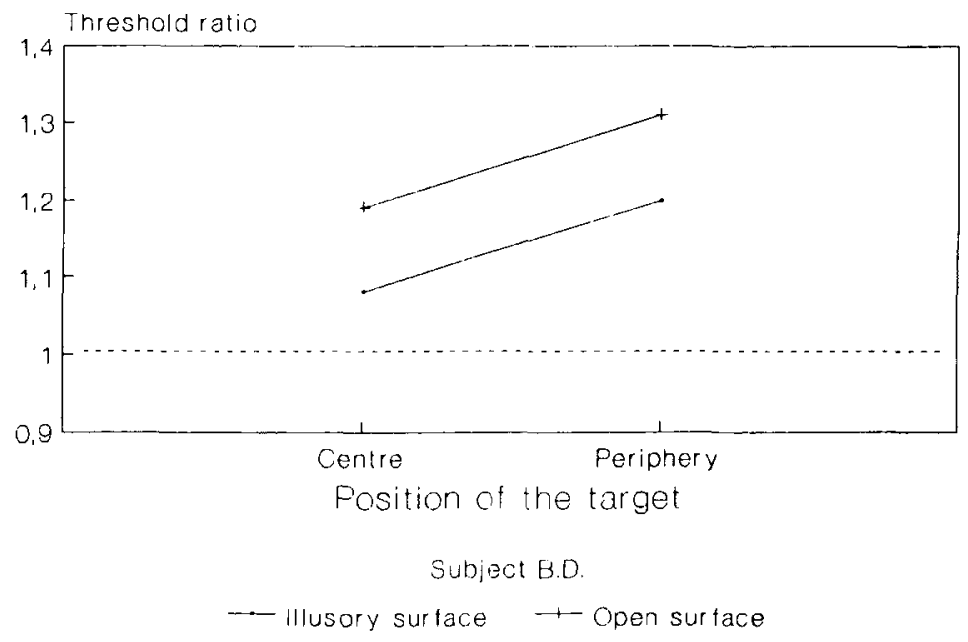

Figure 7. Results obtained with Subject B.D. in Experiment 3. The graphs show that increment threshold ratios (threshold within configuration/threshold on homogeneous background) decrease in the center of configurations with and without global figure enhancement. Coeficients of intraindividual variability do not exceed .04 for any of the conditions. 


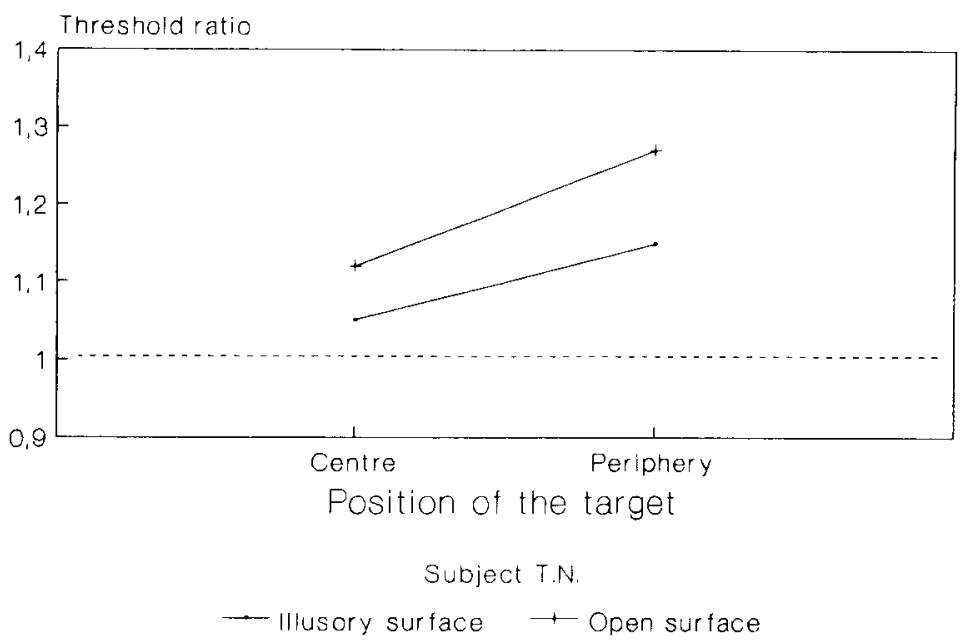

\footnotetext{
Figure 8. Results obtained with Subject T.N. in Experiment 3. The graphs show the same tendencies as those observed with B.D. Coefficients of intraindividual variability do not exceed .03 for any of the conditions.
}

illusory square is visible. This result should be due to the fact that the borders of the contrast-inducing elements in the control figure are closer to the target, for any of its positions. The effects on the increment threshold diminish with increasing distance of the target from the inducing elements in both configurations, the Kanizsa square and the control figure. Data from a previous study (Dresp $\&$ Bonnet, 1991) have shown that the decrease in thresholds is rather progressive in both cases. This is a particularly striking observation, because it shows that local sensitivity varies in the same way, whether a configuration gives rise to the perception of a homogeneously enhanced surface or not.

The results of this experiment and their consistency with earlier findings from the use of nonillusory surfaces (cf. Van Esen \& Novak, 1974) seem to indicate that global brightness or darkness of surfaces is not generated at early stages of processing. The observations reported here lead us to discuss to what extent filling-in assumptions, given their tendency to relate local and global brightness to the same level of processing, provide an entirely satisfactory heuristic for our understanding of an essential aspect of form perception.

\section{GENERAL DISCUSSION}

Global psychophysical measures (Experiments 1 and 2) show that an illusory figure such as the Kanizsa square gives rise to effects similar to those that have been reported earlier in simultaneous contrast experiments (Heinemann, 1955, 1972). The induction effects are essentially determined through parameters such as size and distance of the inducing elements. Figural brightness appears to be related to the luminance of the inducers by a power function. The similarity of these observations with spatial contrast measures (see Wyszecki, 1986) leads to the conclusion that simultaneous brightness/darkness induction and its underlying mechanisms must contribute to the genesis of the illusory phenomena in the Kanizsa square.

Although the results of Experiments 1 and 2 certainly provide to some extent an answer to the questions raised by Brigner and Gallagher (1974) and Frisby and Clatworthy (1975), to say that they tend to confirm the idea that the Kanizsa square is generated through a spreading of lateral inhibitory mechanisms would be to jump to conclusions. Although psychophysical data derived from brightness matches are often directly brought into relation with interactions between neural subsystems signaling brightness and darkness of shapes in the visual cortex (Jung, 1973; Spillmann et al., 1976; Spillmann et al., 1984), one should take into account that they are necessarily influenced by global properties of the stimulus pattern. However, increment thresholds (see Fiorentini, 1972, for a review) essentially reflect responses of the visual system to local stimulations and should be less, if at all, influenced by form characteristics other than local contrast.

The results of Experiment 3 have led us to the conclusion that global brightness or darkness must be generated at a higher stage of processing than the one involving interactions between neural subsystems with on-center offsurround receptive field organization. Evidently, this interpretation implies the assumption that increment threshold variations reflect consequences of such lateral interactions. While the elevations in increment thresholds measured close to the borders of the inducing elements can be regarded as psychophysical correlates of a diffusive mechanism (cf. Dresp \& Bonnet, 1991) of the kind described within neural models of form perception (cf. Grossberg \& Mingolla, 1985; Ullmann, 1984), it appears that the filling in requires more than that, since the thresholds decrease in the center of configurations (e.g., 
the Koffka annulus; see Cornsweet \& Teller, 1965; and the Kanizsa square; see Dresp \& Bonnet, 1991) where phenomenal brightness or darkness is as strong as it is in their periphery. The global brightness or darkness of a closed surface does not seem to be simply the result of mechanisms that process border contrast and then diffuse over wide areas tending to average brightness- or darkness-specific activities within boundaries (real or illusory ones). The assumption that filling in works this way (see, e.g., Grossberg \& Mingolla, 1985) implies that local brightness and surface brightness share the same underlying mechanisms and that the global representation is directly derived from local inputs at the same stage of visual information processing. Consequently, increment thresholds should then be elevated to the same amount at any point within the surface.

In the view suggested here, diffusion and filling in would rather be seen as two qualitatively different processes. Diffusion leads to local brightness or darkness variations as they can be seen across luminance borders (cf. Mach bands and similar phenomena). The result of the diffusive process is visible only when other, more specific information about form is not available in the pattern and a more complex process of perceptual organization is not required. If we were to consider diffusion alone, we would perceive the inner surrounds of a Kanizsa square, or a Koffka annulus, as darker or brighter than the center of the figure. Diffusion, in some sense, would deliver us a "sketch" of an illusory surface if no other process of perceptual organization were to enter into the chain of processing. If we perceive homogeneously enhanced figures, it is because the output of diffusive mechanisms is modified at later stages of processing, giving rise to other, less specific and more complex, perceptual events. Figure-ground segregation has to be considered the principal candidate here. In other words, what we perceive in the case of the Kanizsa square and similar configurations must be the result of an integration of the "sketch" into a global representation of an essential property of form-its brightness or darkness with regard to a general background.

A basic idea within low-level models of form perception is that oriented inputs lead to combinations of signals of shape and luminance (referred to as "boundaryfeature tradeoff' in Grossberg \& Mingolla, 1985, for example) that can trigger various, quantitatively different, diffusive processes. The filling in of closed regions is explained on the basis of the same general principle, and, from a strictly mechanistic point of view, this appears to be a plausible solution. However, empirical evidence from earlier studies and the one presented here seems to indicate that filling in is probably not reducible to a particular kind of diffusive process.

If we consider the systematicity reducible of sensitivity variations within regions close to luminance borders reported in various studies, there can be no doubt that the idea of a diffusive process to account for local brightness or darkness is empirically justified. This does not seem to hold equally for figural brightness or darkness, since it alters sensitivity in a paradoxical way, suggesting a genesis at higher stages by way of holistic processes of perceptual organization. These processes necessarily do integrate the outputs of local mechanisms, but in such a way as to modify them qualitatively. In these respects, it appears that mechanisms that consist of combining inputs via a set of locally operating routines do not always provide entirely satisfactory heuristics for our understanding of how figural properties are generated.

\section{REFERENCES}

Brigner, W. L., \& Gallagher, M. B. (1974). Subjective contour: Apparent depth or simultaneous brightness contrast? Perceptual \& Motor Skills, 38, 1047-1053.

Cornsweet, T. N., Teller, D. Y. (1965). Relation of increment thresholds to brightness and luminance. Journal of the Optical Society of America, 55, 1303-1308.

DRESP B., \& BONNET, C. (1991). Psychophysical evidence for low-level processing of illusory contours and surfaces in the Kanizsa square. Vision Research, 31, 1813-1817.

Dresp, B., Lorenceau J., \& Bonnet, C. (1990). Apparent brightness enhancement in the Kanizsa square with and without illusory contours. Perception, 19, 483-489.

Fiorentini, A. (1972). Mach band phenomena. In D. Jameson \& L. M Hurvich (Eds.), Handbook of sensory physiology: Visual psychophysics (pp. 188-201). New York: Springer

Frisby, J. P., \& Clatworthy, J. L. (1975). Illusory contours: Curious cases of simultaneous brightness contrast? Perception, 4, 349-357.

Gerrits, H. J. M., \&endrik, A. J. H. (1970). Simultaneous contrast, filling-in process and information processing in man's visual system. Experimental Brain Research, 11, 411-430.

GrossberG, S., \& Mingolla, E. (1985). Neural dynamics of form perception: Boundary completion, illusory figures and neon color spreading. Psychological Review, 92, 173-211.

Grossberg, S., \& Mingolla, E. (1987). The role of illusory contours in visual segmentation. In S. Petry \& G. E. Meyer (Eds.). The perception of illusory contours (pp. 116-125). New York: Springer.

HeinemanN, E. G. (1955). Simultaneous brightness induction as a function of inducing and test field luminances. Joumal of Experimental Psychology, 50, 89-96.

HeinemanN, E. G. (1972). Simultaneous brightness induction. In D. Jameson \& L. M. Hurvich (Eds.), Handbook of sensory physiology: Visual psychophysics (pp. 146-169). New York: Springer-Verlag.

JORY, M. (1987). Increment thresholds in illusory contour line patterns. In S. Petry \& G. E. Meyer (Eds.), The perception of illusory contours (pp. 183-189). New York: Springer.

JUNG, R. (1973). Visual perception and neurophysiology. In R. Jung (Ed.), Handbook of sensory physiology (Vol. VII/3A, Pp. 54-58). Berlin: Springer.

KANIzsA, G. (1955). Margini quasi-percettivi in campi con stimolazione omogenea. Rivista di Psicologia, 49, 7-13.

Spillmann, L., Fuld, K., \& Gerrits, H. J. M. (1976). Brightness contrast in the Ehrenstein illusion. Vision Research, 16, 713-719.

Spillmann, L., Fuld, K., \& Neumeyer, C. (1984). Brightness matching, brightness cancellation, and increment threshold in the Ehrenstein illusion. Perception, 13, 512-520.

TYLER, C. W. (1987). Analysis of visual modulation sensitivity: III. Meridional variations in peripheral flicker sensitivity. Journal of the Optical Society of America A, 4, 1612-1619.

Ullmann, S. (1984). Visual routines. Cognition, 18, 97-159.

VAN ESEN, J. S., NovaK, S. (1974). Detection thresholds within a 
display that manifests contour enhancement and brightness contrast. Journal of the Optical Society of America, 64, 726-729.

WatANABE, T., \& OYAMA, T. (1988). Are illusory contours a cause or a consequence of apparent differences in brightness and depth in the Kanizsa square? Perception, 17, 513-521.

WySZECKI, G. (1986). Color appearance. In K. R. Boff, L. Kaufmann,
\& J. P. Thomas (Eds.), Handbook of perception and human perfor mance: Sensory processes and perception (pp. 9-12-9-23) New York: Wiley.

(Manuscript received September 9, 1991; revision accepted for publication May 19, 1992.) 